\title{
Characteristics of severe malaria in hospitalized children in Ho Chi Minh City from 2012 to 2019
}

\author{
Ha, M.T. ${ }^{1}$, Ho, T.A.T. ${ }^{2}$, Nguyen, A.N. ${ }^{1}$, Nguyen, T.A. ${ }^{3 *}$ \\ ${ }^{1}$ University of Medicine and Pharmacy at Ho Chi Minh City, 700000 Ho Chi Minh City, Vietnam \\ ${ }^{2}$ Khanh Hoa General Hospital, Nha Trang City, 650000 Khanh Hoa Province, Vietnam \\ ${ }^{3}$ Molecular Biomedical Center, University Medical Center - Campus 2, 700000 Ho Chi Minh City, Vietnam \\ *Corresponding author: anh.nt@umc.edu.vn
}

\section{ARTICLE HISTORY}

Received: 3 June 2021

Revised: 26 July 2021

Accepted: 26 July 2021

Published: 30 August 2021

\begin{abstract}
In Vietnam, severe malaria is currently rare but is a life-threatening disease. It may be misdiagnosed with other common diseases. This descriptive study aimed to characterize severe malaria and its clinical aspects, as well as outcomes of infected pediatric patients to improve case management. The case-series study was carried out based on medical records of children aged between one month and 15 years with malaria diagnosed by blood smear or rapid diagnostic test. Chi-squared test with the $p$ values less than 0.05 were considered statistically significant. There were 47 cases enrolled in the study. The prevalence of severe malaria was $29.8 \%$ ( $57.1 \%$ in children under five). The morbidity was $71.4 \%$ in male and $28.6 \%$ in female. Common clinical signs of severe malaria were fever $(100 \%)$, severe anemia (21.4\%), hepatomegaly (85.7\%), and splenomegaly (71.4\%). Common biological abnormalities in severe malaria were anemia, thrombocytopenia, increased liver enzymes, and high CRP level. The severe malaria was mainly caused by P. falciparum (100\%). The age range for those infected with $P$. falciparum was $6.5 \pm 4.5$ years (min 0.3; $\max 14.9$ ). The successful rate of treatment was $92.9 \%$ with artesunate. Antimalarial treatment time was $9.0(6-12)$ days for severe malaria, which was twice as many as that for non-severe malaria $(p=0.067)$. The current clinical and biological findings of severe malaria are different from those in previous times, which make it easy to be overlooked. Therefore, it's important to perform malaria diagnostic tests when there're clinical suggestions of severe malaria, including fever, hepatomegaly or splenomegaly.
\end{abstract}

Keywords: Plasmodium sp.; pediatric; severe malaria; Vietnam.

\section{INTRODUCTION}

Malaria is a life-threatening parasitic disease caused by Plasmodium sp., which are transmitted to humans through the bite of infected female Anopheles mosquitoes. According to the World Health Organization (WHO), in 2018, an estimated 228 million cases of malaria occurred worldwide and there were an estimated 405000 deaths from malaria globally (WHO, 2019). Despite recent advances in preventive and therapeutic approaches, as well as improvements in socioeconomic conditions, children aged less than 5 years are the most vulnerable group affected by malaria. In 2018, they accounted for $67 \%$ (272 000) of all malaria deaths worldwide (WHO, 2019). In Vietnam, the rates of morbidity and mortality associated with malaria in recent years have declined dramatically. There were 1422 cases of malaria and one death from malaria in 2021, compared with more than 1 million cases and 4646 deaths in 1991 (Quy Nhon IMPE, 2021). However, severe malaria, a complicated malaria infection status because of the latency in detection and the misdiagnosis of other diseases, is worth of being noticed, especially in Vietnam (VMoH, 2016). This study aimed to describe the clinical and biological characteristics of Vietnamese pediatric patients with severe malaria. These can be useful to provide warnings and contribute to the early detection of and control this dangerous disease.

\section{MATERIALS AND METHODS}

\section{Study population}

The study population included one month to 15 year old children who were admitted to Children Hospital 1 and Children Hospital 2 at Ho Chi Minh City from January 2012 to January 2019, with malaria confirmed by thick blood smear (TBS) and/or a rapid diagnostic test (RDT) (VMoH, 2016).

The malaria parasite density is estimated by Giemsastained thick blood films on a glass slide (Tangpukdee et al., 2009). The exact range of malaria parasite density for,+++ , 
+++ and ++++ score were 1 - 10 parasite(s)/100 fields, $11-100$ parasites $/ 100$ fields, $1-10$ parasite(s)/field, and $11-100$ parasites/field respectively. Thin blood films were used for species' confirmation of the presenting malaria parasites comprising Plasmodium falciparum, Plasmodium vivax, Plasmodium malariae, Plasmodium ovale, Plasmodium knowlesi, trophozoite, schizont and gametocyte. The rapid diagnostic test was SD BIOLINE Malaria Ag P.f test (Abbott).

\section{Study design}

In this case series, patients were identified from the list of patients who discharged with ICD 10 code of malaria (B50.9). Data including the epidemiological, clinical and biological characteristics, patient progression, treatment and final outcome were extracted after reviewing medical records of patients who met the above criteria for malaria diagnosis. All eligible patients were included, except for patients with insufficient information or having comorbidities. Severe malaria is defined by clinical or biological diagnostic criteria in the Ministry of Health Vietnam guidelines for diagnosis, treatment and prevention of malaria ( $\mathrm{VMoH}, 2016)$, adopted from WHO guidelines (WHO, 2012). The signs of severe malaria are:

Clinical signs: Consciousness disorders (Blantyre coma scale $<5$ points), extreme fatigue (unable to sit, stand or walk around without support), convulsions (2 times/24 hours), deep breath with $>20$ times/minutes and disordered breathing, acute pulmonary edema, acute respiratory distress (shortness of breath, $\mathrm{SpO}_{2}<92 \%$ ), circulatory failure or shock (rapid and weak pulses, systolic blood pressure reduces 20 $\mathrm{mmHg}$ from normal blood pressure of children, cold limbs, oliguria), acute renal failure (amount of urine $<0.5 \mathrm{ml} / \mathrm{kg}$ / hours), jaundice, abnormal bleeding (under the skin, in muscle, in the intestines or at the site of injection; prolonged bleeding; black stools; vomiting blood).

Biological signs: hyperparasitemia (parasitemia $>10 \%$ of infected red blood cells), hypoglycemia (glycemia $<70 \mathrm{mg} / \mathrm{dl}$ or $<4 \mathrm{mmol} / \mathrm{l}$ ) or severe hypoglycemia (glycemia $<50 \mathrm{mg} / \mathrm{dl}$ or $<2.7 \mathrm{mmol} / \mathrm{l}$ ), blood sugar/serum $<2.2 \mathrm{mmol} / \mathrm{l}$ or $<40 \mathrm{mg} /$ $\mathrm{dl}$, metabolic acidosis $\mathrm{pH}<7.35$ (serum bicarbonates $<15$ $\mathrm{mmol} / \mathrm{l})$, severe anemia $(\mathrm{Hb}<5 \mathrm{~g} / \mathrm{dl}$ or hematocrit $<15 \%)$, hemoglubinuria, increased serum lactate (lactate $>4 \mathrm{mmol} /$ I), kidney failure (serum creatinine $>3 \mathrm{mg} \%$ or $>265 \mu \mathrm{mol} / \mathrm{l}$; or blood ure $>20 \mathrm{mmol} / \mathrm{l}$ ), serum bilirubin $>50 \mathrm{imol} / \mathrm{l}$ or $3 \mathrm{mg} /$ $\mathrm{dl}$, and chest X-ray has blurred image of 2 hilum and bottom of lung.

\section{Treatment}

After admission, the antimalarial drug regimen was used as recommended by the WHO in 2012 (WHO, 2012) and adopted by the Ministry of Health Vietnam in 2016 (VMoH, 2016). For malaria cases caused by P. falciparum, artemisininpiperaquine were used and continued with primaquine if pediatric patients were over 6 months. For cases caused by $P$. vivax, chloroquine was used and combined with primaquine in case of patients over 6 months. For severe malaria, intravenous artesunate was used firstly and then changed to oral artemisinin-piperaquine as normal malaria. Primaquine was added to the regimen if patients were over 6 months.

\section{Data analysis}

Categorical and continuous variables were described by frequency (percentage) and median (range), respectively. Proportions were compared by Chi-squared test or Fisher's Exact test when more than $20 \%$ of cells have expected frequencies $<5$, while the differences between groups were detected by the Mann-Whitney test. Statistical significance was declared when two-sided p-value was less than 0.05 . Data were processed using SPSS Statistics for Windows, version 16.0 (Chicago, SPSS Inc.).

\section{RESULTS}

\section{General description of study population}

Over seven years of following-up (2012 - 2019), 47 malaria cases were eligible for the analysis. Fourteen cases were classified as severe malaria, giving a percentage of $29.8 \%$ $(14 / 47)$. The number of males with severe malaria $(10 / 14$; $71.4 \%$ ) was nearly 3 times higher than the number of females with severe malaria $(4 / 14 ; 28.6 \%)$ in this study. Number of patients with normal nutritional status was nearly sesquialteral compared to number of patients with malnutrition (eight vs. six). Time from disease onset to admission was shorter in severe cases. Almost patients came from South-East and Middle-Highland regions of Vietnam. Only one of patients came from Ho Chi Minh City (Table 1).

Table 1. General characteristics of study population

\begin{tabular}{|c|c|c|c|c|}
\hline \multirow{2}{*}{ Characteristics } & \multicolumn{2}{|c|}{ Severe malaria } & \multirow{2}{*}{ Total $(n=47)$} & \multirow{2}{*}{$p$ value } \\
\hline & Yes $(n=14)$ & No $(n=33)$ & & \\
\hline \multicolumn{5}{|l|}{ Sex } \\
\hline Males & $71.4 \%(10 / 14)$ & $57.5 \%(19 / 33)$ & $61.7 \%(29 / 47)$ & \multirow{2}{*}{0.516} \\
\hline Females & $28.6 \%(4 / 14)$ & $42.4 \%(14 / 33)$ & $38.3 \%(18 / 47)$ & \\
\hline \multicolumn{5}{|l|}{ Age (years) } \\
\hline$\leq 5$ & $57.1 \%(8 / 14)$ & $30.3 \%(10 / 33)$ & $38.3 \%(18 / 47)$ & \multirow{2}{*}{0.083} \\
\hline$>5$ & $42.9 \%(6 / 14)$ & $69.7(23 / 33)$ & $61.7 \%(29 / 47)$ & \\
\hline \multicolumn{5}{|l|}{ Nutritional status by weight } \\
\hline Normal & $57.1 \%(8 / 14)$ & $69.7 \%(23 / 33)$ & $66.0 \%(31 / 47)$ & \multirow{3}{*}{0.495} \\
\hline Malnutrition & $42.9 \%(6 / 14)$ & $27.3 \%(9 / 33)$ & $31.9 \%(15 / 47)$ & \\
\hline Obesity & $0 \%(0 / 14)$ & $3.0 \%(1 / 33)$ & $2.1 \%(1 / 47)$ & \\
\hline Days from disease onset to admission & $7.0(5-9)$ & $10(6-15)$ & $8(5-14)$ & 0.199 \\
\hline \multicolumn{5}{|l|}{ Residency } \\
\hline Middle - Highland region & $50.0 \%(7 / 14)$ & $42.4 \%(14 / 33)$ & $44.7 \%(21 / 47)$ & \multirow{3}{*}{0.888} \\
\hline South - East region & $42.9 \%(6 / 14)$ & $48.5 \%(16 / 33)$ & $46.8 \%(22 / 47)$ & \\
\hline Ho Chi Minh City & $7.1 \%(1 / 14)$ & $9.1 \%(3 / 33)$ & $8.5 \%(4 / 47)$ & \\
\hline
\end{tabular}




\section{Clinical and biological characteristics}

Our study showed that all patients had fever, but fever $\geq 39^{\circ} \mathrm{C}$ accounted for only $35.7 \%$ (5/14) patients, and the fever was not typical for periodic fever. In addition, patients with severe malaria presented diverse symptoms, from gastrointestinal disorders to headache, altered consciousness, jaundice, anemia, hepatomegaly and splenomegaly; but the most important symptoms were hepatomegaly and splenomegaly (71.4\%; 10/14). However, there was no statistically significant difference in clinical symptoms between patients with and without severe malaria $(p>0.05)$ (Table 2$)$.

In the results on completed blood counts of patients with severe malaria showed that the leukocyte counts were almost within normal ranges $(p=0.538)$. All patients had moderate to severe anemia; however, $\mathrm{Hb}<5 \mathrm{~g} / \mathrm{dL}$ in only $6.4 \%$ of cases especially $(p=0.189)$. Most platelet counts were less than $100.000 / \mathrm{mm}^{3}(p=0.033)$. There was a significant reduction in monocyte count in severe malaria cases $\left(0.4 \times 10^{3} / \mathrm{mm}^{3}\right)$ compared to non-severe malaria cases $\left(0.6 \times 10^{3} / \mathrm{mm}^{3}\right)(p=0.025)$, as well as in Eosinophil count between the two groups $\left(0.02 \times 10^{3} / \mathrm{mm}^{3}\right.$ vs. $0.11 \times 10^{3} / \mathrm{mm}^{3}$; $p=0.024)$ (Table 2).

\section{Type and density of malaria parasites}

The main cause of severe malaria was P. falciparum $(100 \%$, $12 / 12$ ), and the density of parasites in peripheral blood was often high and higher in severe malaria. There was a statistical difference in types $(p=0.019)$ as well as density $(p=0.016)$ of parasites between severe and non-severe malaria group (Table 3 ).

\section{Time to treatment response}

Of the 47 children enrolled in the study, we noted that $83 \%$ (39/47) of the children did not have enough information of treatment prior to admission. $17 \%(8 / 47)$ of the children received treatment before there admission. Amongst the drugs taken, $2.1 \%(1 / 47) ; 4.3 \%(2 / 47)$ and $10.6 \%(5 / 47)$ of the patients took artemisinin-piperaquine, primaquinechloroquine, and artesunate respectively as recorded.

In our patients, $57.5 \%(27 / 47)$ of the patients took artemisinin-piperaquine, together with primaquine (10/27) and chloroquine-primaquine (1/27). $14.9 \%$ (7/47) of the patients took chloroquine-primaquine, and continued with primaquine after discharged. $27.7 \%$ (13/47) of the patients

Table 2. Clinical and biological findings in severe and non-severe malaria patients

\begin{tabular}{|c|c|c|c|}
\hline \multirow{2}{*}{ Symptoms } & \multicolumn{2}{|c|}{ Severe malaria } & \multirow{2}{*}{$\mathrm{p}$} \\
\hline & Yes $(n=14)$ & No $(n=33)$ & \\
\hline \multicolumn{4}{|l|}{ Clinical findings } \\
\hline Fever $\geq 39^{\circ} \mathrm{C}$ & $35.7 \%(5 / 14)$ & $15.2 \%(5 / 33)$ & 0.115 \\
\hline \multicolumn{4}{|l|}{ Type of fever } \\
\hline Periodic fever & $50.0 \%(7 / 14)$ & $78.6 \%(22 / 28)$ & 0.059 \\
\hline Continuous fever & $50.0 \%(7 / 14)$ & $21.4 \%(6 / 28)$ & \\
\hline Duration of fever (days) & $9.5(7.0-12.0)$ & $11(8.0-17.0)$ & 0.383 \\
\hline Gastrointestinal disorders & $35.7 \%(5 / 14)$ & $27.3 \%(9 / 33)$ & 0.563 \\
\hline Headache & $8.3 \%(1 / 12)$ & $19.4 \%(6 / 31)$ & 0.652 \\
\hline Altered consciousness & $14.3 \%(2 / 14)$ & $0 \%(0 / 33)$ & 0.084 \\
\hline Jaundice & $14.3 \%(2 / 14)$ & $6.1 \%(2 / 33)$ & 0.572 \\
\hline Severe anemia & $21.4 \%(3 / 14)$ & $3.0 \%(1 / 33)$ & 0.073 \\
\hline Seizure & $14.3 \%(2 / 14)$ & $6.1 \%(2 / 33)$ & 0.572 \\
\hline Hepatomegaly & $85.7 \%(12 / 14)$ & $72.7 \%(24 / 33)$ & 0.464 \\
\hline Splenomegaly & $71.4 \%(10 / 14)$ & $60.6 \%(20 / 33)$ & 0.529 \\
\hline Hepatomegaly and splenomegaly & $71.4 \%(10 / 14)$ & $57.6 \%(19 / 33)$ & 0.561 \\
\hline \multicolumn{4}{|l|}{ Biological findings } \\
\hline \multicolumn{4}{|l|}{ Completed blood count } \\
\hline Leukocytes $\left(1.000 / \mathrm{mm}^{3}\right)$ & $5.3(4.2-8.8)$ & $6.5(5.1-7.7)$ & 0.538 \\
\hline Hemoglobin $(\mathrm{g} / \mathrm{dL})$ & $7.7(6.5-9.0)$ & $8.8(7.5-10.6)$ & 0.189 \\
\hline Platelet count $\left(1.000 / \mathrm{mm}^{3}\right)$ & $69.5(52-131)$ & $136(82.5-187)$ & 0.033 \\
\hline Monocyte $\left(1.000 / \mathrm{mm}^{3}\right)$ & $0.4(0.2-0.6)$ & $0.6(0.4-0.9)$ & 0.025 \\
\hline Neutrocytes $\left(1.000 / \mathrm{mm}^{3}\right)$ & $2.7(2.3-4.1)$ & $2.8(1.7-3.5)$ & 0.642 \\
\hline Lymphocytes $\left(1.000 / \mathrm{mm}^{3}\right)$ & $2.1(0.9-3.5)$ & $2.4(1.5-3.5)$ & 0.515 \\
\hline Eosinophils $\left(1.000 / \mathrm{mm}^{3}\right)$ & $0.02(0.02-0.06)$ & $0.11(0.03-0.20)$ & 0.024 \\
\hline \multicolumn{4}{|l|}{ Blood Biochemistry } \\
\hline $\mathrm{CRP}(\mathrm{mg} / \mathrm{L})$ & $117.8(70.0-143.0)$ & $63.3(34.0-110.0)$ & 0.025 \\
\hline AST $>40 \mathrm{U} / \mathrm{L}$ & $78.6 \%(11 / 14)$ & $30.3 \%(10 / 33)$ & 0.004 \\
\hline $\mathrm{ALT}>40 \mathrm{U} / \mathrm{L}$ & $71.4 \%(10 / 14)$ & $9.1 \%(3 / 33)$ & $<0.001$ \\
\hline Total Bilirubin $\geq 1.5 \mathrm{mg} / \mathrm{dL}$ & $7.1 \%(1 / 13)$ & $3.7 \%(1 / 27)$ & 1.000 \\
\hline \multicolumn{4}{|l|}{ Urine } \\
\hline Hemoglobinuria & $30.0 \%(3 / 10)$ & $5.3 \%(1 / 19)$ & 0.105 \\
\hline Creatinine $\geq 2 \mathrm{mg} / \mathrm{dL}$ & $7.1 \%(1 / 13)$ & $0 \%(0 / 33)$ & 0.298 \\
\hline
\end{tabular}


Table 3. Type and density of parasite in severe and non-severe malaria patients

\begin{tabular}{|c|c|c|c|}
\hline & \multicolumn{2}{|c|}{ Severe malaria } & \multirow{2}{*}{$p$} \\
\hline & Yes $(n=14)$ & No $(n=33)$ & \\
\hline \multicolumn{4}{|l|}{ Plasmodium species } \\
\hline P. falciparum & $100 \%(12 / 12)$ & $64.5 \%(20 / 31)$ & $0.019^{*}$ \\
\hline P. vivax & $0 \%(0 / 12)$ & $35.5 \%(11 / 31)$ & \\
\hline P. falciparum and $P$. vivax & $0 \%(0 / 14)$ & $3.0 \%(1 / 33)$ & \\
\hline Undetermined & $14.3 \%(2 / 14)$ & $3.0 \%(1 / 33)$ & \\
\hline \multicolumn{4}{|l|}{ Density of parasite } \\
\hline+ & $25.0 \%(3 / 12)$ & $68.8 \%(22 / 32)$ & 0.016 \\
\hline,+++++ and ++++ & $75 \%(9 / 12)$ & $31.2 \%(10 / 32)$ & \\
\hline
\end{tabular}

${ }^{*}$ Co-infection and undertemined parasites were not included in the statistical analysis.

Table 4. Time to treatment response in severe and non-severe malaria patients

\begin{tabular}{lcc}
\hline & & Severe malaria \\
\cline { 2 - 3 } Time (mean, range) & Yes (n=14) & No (n=33) \\
\hline Days to fever clearance & $2(1-3)$ & $2(1-2)$ \\
Days to parasite clearance after treatment & $4(3-7)$ & $3(3-4)$ \\
Duration of antimalarial treatment (days) & $9(6-12)$ & $4(3-7)$ \\
Length of hospital stay (days) & $11(9-15)$ & 9.262 \\
\hline
\end{tabular}

took artesunate, together with artemisinin-piperaquine (7/13) and artemisinin-piperaquine-primaquine (5/13).

Patients with severe malaria were mainly treated with artesunate, with the success rate of $13 / 14$ (92.9\%). Death was caused by hemophagocytic syndrome. Time to fever clearance and undetectable parasite on peripheral blood smear was short, with a median of 2 and 4 days, respectively. However, the duration of antimalarial treatment and length of hospital stay were long with medians of 9 and 11 days, correspondingly. There was no difference in time to fever clearance, parasite clearance, and length of hospital stay between severe and non-severe malaria patients $(p>0.05)$. However, duration of antimalarial treatment was twice as long in the severe malaria group $(p=0.067)$ (Table 4).

\section{DISCUSSION}

Severe malaria is a life-threatening disease as it can cause multiple-organ dysfunctions as well as metabolic disorders. Delays in detection and treatment of this condition can be fatal. Therefore, early detection and appropriate management have an important role in managing severe malaria.

The severe malaria discovered in this study was similar to the finding $(29.2 \%)$ by Chiabi et al. in Cameroon in 2007 (Chiabi et al., 2020), but higher compared to other studies of the same period of time such as Geleta et al. in Ethiopia in 2015 (25.7\%) (Geleta \& Ketema, 2016) and Edelu et al. in Nigeria in 2018 (5.5\%) (Edelu et al., 2018). The newest study from Cameroon also conducted by Chiabi et al. in 2020 showed a lower incidence of $26.15 \%$ in the same settings. Despite the fact that these studies were different in geographical areas and sample sizes, the high incidence rate of severe malaria cases in our study could be explained for several reasons. Firstly, majority of cases was constituted by non- severe malaria in our data set. The sampling method was therefore unlikely to be the cause of high incidence rate. Secondly, our study was conducted in the two biggest tertiary pediatric hospitals from the Southern of Vietnam which usually admitted severe patients from Ho Chi Minh and nearby provinces. Thirdly, it could be due to delayed treatment because of misdiagnosing with other diseases and/or delayed detecting of malaria parasites. The misdiagnosis might be due to the low incidence rate of malaria in Vietnam. Clinical doctors could think of other common diseases at admission such as Dengue fever. Therefore, suspected malaria cases had been omitted at the beginning until there were severe signs of malaria infection. That's the reason why it suggested that for fever cases with abnormal clinical symptoms such as anemia, hepatomegaly and splenomegaly, blood test looking for malaria parasites should be carried out to avoid misdiagnosis.

The prevalence of severe malaria in males was higher than in females in this study, which has been observed in previous studies (Mockenhaupt et al., 2004; Dzeing-Ella et al., 2005; Al-Taiar et al., 2006; Orimadegun et al., 2007; Edelu et al., 2018). The exact reason why male is more affected is currently unknown; however, there is a suggestion of genetic influences and sex hormones (Zuk \& McKean, 1996). There was no difference in age, nutritional status and residence location between general malaria and severe malaria.

Regarding the age of severe malaria infection, children under 5 years of age are more susceptible than those over 5 years old. This findings was in line with WHO, which states that children less than 5 years are the most vulnerable group to malaria (WHO, 2019). This pattern has also been seen in other studies, and the explanation could be that younger children have weak immunity to malaria, which can easily lead to severe malaria and complications (Mockenhaupt et 
al., 2004; Dzeing-Ella et al., 2005; Al-Taiar et al., 2006; Edelu et al., 2018). This study noted that nutritional status among many patients with severe malaria was normal (57.1\%). This is also demonstrated in previous studies and the authors concluded that nutrition factors are not determinants of severe malaria in children (Koram et al., 1995; Kateera et al., 2015).

In addition, our study showed that only $46.8 \%$ of cases were diagnosed with malaria at admission. Even though treatment is vital during the first 24 hours (Schumacher and Spinelli, 2012), the rest of cases in our study only received confirmed diagnosis and initiated treatment post $2-3$ days of hospitalization for the misdiagnosed patients. The most common misdiagnosis at admission was Dengue fever, especially during the Dengue fever outbreak season. Therefore, it is suggested to include conducting malaria diagnostic tests towards children presenting fever with unusual clinical symptoms from endemic regions for malaria.

Among these patients, 6 cases were cerebral malaria $(42.8 \%)$, and the remaining cases had moderate to severe anemia, including 3 cases of severe anemia, 4 cases of acute hemolysis causing hemoglobinuria and 1 case had extremely high density of malaria parasite. This composition was different from the previous periods when cerebral malaria was the most common type of severe malaria $(80-85 \%)$ (Edelu et al., 2018). The shift from cerebral malaria to severe anemia or hemolysis as the most common presentation of severe malaria is a worthy notice in the current clinical setting.

Regarding clinical symptoms of severe malaria, our results suggested that in addition to established clinical criteria for differentiating severe and non-severe malaria, other clinical symptoms did not have much added value. This has also been noted in previous studies (Edelu et al., 2018). Anemia is a common problem in malaria and severe malaria because of erythrocyte destruction by parasite (Haldar \& Mohandas, 2009; Sumbele et al., 2016; White, 2018). In addition, this study also demonstrated a significant reduction in platelet counts in patients with severe malaria. Thrombocytopenia is known to be associated with high parasite density in severe malaria, causing platelets to be aggregated in the spleen and peripheral blood (Ladhani et al., 2002). Therefore, thrombocytopenia is one of the recommended signs of severe malaria, and it must be considered in assessing malaria patients. The decrease in monocyte count in patients with severe malaria has been demonstrated in previous studies (Chiwakata et al., 2000; Ladhani et al., 2002). Monocytes are important in developing immunity against malaria through the phagocytosis of infected red blood cells (Tebo et al., 2001). The reduction in monocyte may reduce the body's ability to protect against malaria parasites, and lead to severe malaria and complications. Therefore, the reduction in monocyte can be seen as a risk factor of severe outcome in children with malaria (Ladhani et al., 2002).

The majority of patients with severe malaria had hepatocyte damage characterized by increased liver enzymes and CRP while changes in renal functions were insignificant. Hemoglobinuria occurred in 4 patients. The elevation of liver enzymes in patients with severe malaria was significantly higher than in patients with non-severe malaria. In addition, level of CRP in patients' blood with severe malaria was much higher, which can be explained by the increased inflammatory response in severe malaria (Kochar et al., 2010). Statistical comparisons suggested patients with severe malaria had significantly lower platelet count in peripheral blood and higher chance to have increased liver enzymes than patients with general malaria $(p<0.05)$.

In this study, $P$. falciparum was the main cause of malaria and severe malaria, which is consistent with a previous study, especially when the density of malaria parasites in the blood increases (WHO, 2019). Therefore, clinical attention should be paid to patients with $P$. falciparum malaria and high parasite density in blood, which suggests that severe malaria is likely to occur. However, a recent study has noted that $P$. vivax may also be associated with severe malaria and death (Kochar et al., 2010).

Our study shows a success rate of $92.9 \%$ on artesunate, with both time-to-fever-clearance and time-to-parasiteclearance on peripheral blood had median of 2 and 4 days respectively. This suggests that the parasite is still susceptible to artesunate. Even though there was no statistically significant difference between severe and non-severe malaria in these measures, the duration of antimalarial drug treatment in patients with severe malaria was significantly longer than in non-severe malaria patients. In clinical practice, cases with severe malaria are usually initiated on slow intravenous artesunate, then switched to oral therapy based on clinical improvement, and the oral therapy was continued until the patient was completely stable. Therefore, the duration of antimalarial drug treatment is usually longer than that of non-severe malaria patients. There was only one case of treatment failure due to hemophagocytic syndrome, and although the parasites had been cleared, the disease still progressed to multiorgan failure and resulted in death. In general, noncomplicated and severe malaria still respond well to the antimalarial drug artesunate. In order to effectively managing patients with severe malaria, it is important to detect severe malaria and use antimalarial drugs early.

The strength was that our data spanned a relatively long period of time ( 7 years) from the two largest pediatric hospitals in Ho Chi Minh City. Despite the fact that this study had a small sample size, it involved both complicated and uncomplicated malaria cases. Such information helped evaluate the effectiveness of artesunate, an antimalarial drug prescribed for severe malaria.

\section{CONCLUSIONS}

The presenting results suggest that clinical and biological characteristics of severe malaria have changed. Therefore, it is vital to perform malaria diagnostic tests when there are clinical suggestions such as fever accompanied with anemia, hepatomegaly or splenomegaly. Starting with antimalarial drug artesunate early is still an important approach to improve prognosis.

\section{ACKNOWLEDGMENTS}

We deeply thank Children's Hospital 1 and Children's Hospital 2 in Ho Chi Minh City for providing data for the study.

\section{Conflict of Interests}

The authors declare that they have no conflict of interests. 


\section{REFERENCES}

Al-Taiar, A., Jaffar, S., Assabri, A., Al-Habori, M., Azazy, A., AlMahdi, N., Ameen, K., Greenwood, B.M. \& Whitty, C.J. (2006). Severe malaria in children in Yemen: two site observational study. British Medical Journal 333: 827. https://doi.org/10.1136/bmj.38959.368819.BE

Chiabi, A., Djimafo, A.N.M., Nguefack, S., Mah, E., Nguefack, D.F. \& Angwafo, F. (2020). Severe malaria in Cameroon: Pattern of disease in children at the Yaounde GynaecoObstetric and Pediatric hospital. Journal of Infection and Public Health 13: 1469-1472. https://doi.org/10.1016/ j.jiph.2020.02.038

Chiwakata, C.B., Hemmer, C.J. \& Dietrich, M. (2000). High levels of inducible nitric oxide synthase mRNA are associated with increased monocyte counts in blood and have a beneficial role in Plasmodium falciparum malaria. Infection and Immunity 68: 394-399. https://doi.org/10.1128/iai. 68.1.394-399.2000

Dzeing-Ella, A., Nze Obiang, P.C., Tchoua, R., Planche, T., Mboza, B., Mbounja, M., Muller-Roemer, U., Jarvis, J., Kendjo, E., Ngou-Milama, E. et al. (2005). Severe falciparum malaria in Gabonese children: clinical and laboratory features. Malaria Journal 4: 1. https://doi.org/10.1186/1475-2875-4-1

Edelu, B.O., Ndu, I.K., Igbokwe, O.O. \& Iloh, O.N. (2018). Severe falciparum malaria in children in Enugu, South East Nigeria. Nigerian Journal of Clinical Practice 21: 13491355.

Geleta, G. \& Ketema, T. (2016). Severe malaria associated with Plasmodium falciparum and Plasmodium vivax among children in Pawe Hospital, North West Ethiopia. Malaria Research Treatment 2016: 1240962. https://doi.org/10.1155/ 2016/1240962

Haldar, K. \& Mohandas, N. (2009). Malaria, erythrocytic infection, and anemia. Hematology. American Society of Hematology. Education Program 2009: 87-93. https://doi.org/ 10.1182/asheducation-2009.1.87

Kateera, F., Ingabire, C.M., Hakizimana, E., Kalinda, P., Mens, P.F., Grobusch, M.P., Mutesa, L. \& van Vugt, M. (2015). Malaria, anaemia and under-nutrition: three frequently co-existing conditions among preschool children in rural Rwanda. Malaria Journal 14: 440. https://doi.org/10.1186/ s12936-015-0973-z

Kochar, D.K., Tanwar, G.S., Khatri, P.C., Kochar, S.K., Sengar, G.S., Gupta, A., Kochar, A., Middha, S., Acharya, J., Saxena, V. et al. (2010). Clinical features of children hospitalized with malaria study from Bikaner, northwest India. The American Journal of Tropical Medicine and Hygiene 83: 981-989. https://doi.org/10.4269/ajtmh.2010.09-0633

Koram, K.A., Bennett, S., Adiamah, J.H. \& Greenwood, B.M. (1995). Socio-economic determinants are not major risk factors for severe malaria in Gambian children. Transactions of the Royal Society of Tropical Medicine and Hygiene 89: 151-154. https://doi.org/10.1016/0035-9203(95)90472-7
Ladhani, S., Lowe, B., Cole, A.O., Kowuondo, K. \& Newton, C.R. (2002). Changes in white blood cells and platelets in children with falciparum malaria: relationship to disease outcome. British Journal of Haematology 119: 839-847. https://doi.org/10.1046/j.1365-2141.2002.03904.x

Mockenhaupt, F.P., Ehrhardt, S., Burkhardt, J., Bosomtwe, S.Y., Laryea, S., Anemana, S.D., Otchwemah, R.N., Cramer, J.P., Dietz, E., Gellert, S. et al. (2004). Manifestation and outcome of severe malaria in children in northern Ghana. The American Journal of Tropical Medicine and Hygiene 71: 167-172. https://doi.org/10.4269/AJTMH.2004.71.167

Orimadegun, A.E., Fawole, O., Okereke, J.O., Akinbami, F.O. \& Sodeinde, O. (2007). Increasing burden of childhood severe malaria in a Nigerian tertiary hospital: implication for control. Journal of Tropical Pediatrics 53: 185-189. https://doi.org/10.1093/tropej/fmm002

Quy Nhon Institute of Malariology, Parasitology and Entomology. (2021). Internal report, 31/3/2021. www.impeqn.org.vn

Schumacher, R.F. \& Spinelli, E. (2012). Malaria in children. Mediterranean Journal of Hematology and Infectious Diseases 4: e2012073. https://doi.org/10.4084/MJHID.2012.073

Sumbele, I.U., Sama, S.O., Kimbi, H.K. \& Taiwe, G.S. (2016). Malaria, moderate to severe anaemia, and malarial anaemia in children at presentation to hospital in the mount Cameroon area: A cross-sectional study. Anemia 2016: 5725634. https://doi.org/10.1155/2016/5725634

Tangpukdee, N., Duangdee, C., Wilairatana, P. \& Krudsood, S. (2009). Malaria diagnosis: a brief review. The Korean Journal of Parasitology 47: 93-102. https://doi.org/10.3347/kjp.2009. 47.2.93

Tebo, A.E., Kremsner, P.G. \& Luty, A.J. (2001). Plasmodium falciparum: a major role for IgG3 in antibody-dependent monocyte-mediated cellular inhibition of parasite growth in vitro. Experimental Parasitology 98: 20-28. https://doi.org/ 10.1006/expr.2001.4619

Vietnam Ministry of Health. (2016). Guidelines for diagnosis, treatment and prevention of malaria. Decision No. 4845/ QĐ-BYT, 8/9/2016.

White, N.J. (2018). Anaemia and malaria. Malaria Journal 17: 371. https://doi.org/10.1186/s12936-018-2509-9

World Health Organization. (2012). Management of severe malaria. A practical handbook. Third edition; 2012. Geneva.

World Health Organization. (2019). World malaria report 2019. World Health Organization. https://apps.who.int/iris/ handle/10665/330011.

Zuk, M. \& McKean, K.A. (1996). Sex differences in parasite infections: patterns and processes. International Journal for Parasitology 26: 1009-1023. https://doi.org/10.1016/ S0020-7519(96)80001-4 